\title{
Analysis of Load-Carrying Capacity for Redundant Free-Floating Space Manipulators in Trajectory Tracking Task
}

\author{
Qingxuan Jia, Yong Liu, Gang Chen, Hanxu Sun, and Junjie Peng \\ Automation School, Beijing University of Posts and Telecommunications, 10 Xitucheng Road, Haidian District, Beijing 100876, China \\ Correspondence should be addressed to Yong Liu; buptly@126.com
}

Received 13 October 2014; Accepted 11 November 2014; Published 8 December 2014

Academic Editor: Yoshinori Hayafuji

Copyright ( 2014 Qingxuan Jia et al. This is an open access article distributed under the Creative Commons Attribution License, which permits unrestricted use, distribution, and reproduction in any medium, provided the original work is properly cited.

\begin{abstract}
The aim of this paper is to analyze load-carrying capacity of redundant free-floating space manipulators (FFSM) in trajectory tracking task. Combined with the analysis of influential factors in load-carrying process, evaluation of maximum load-carrying capacity (MLCC) is described as multiconstrained nonlinear programming problem. An efficient algorithm based on repeated line search within discontinuous feasible region is presented to determine MLCC for a given trajectory of the end-effector and corresponding joint path. Then, considering the influence of MLCC caused by different initial configurations for the starting point of given trajectory, a kind of maximum payload initial configuration planning method is proposed by using PSO algorithm. Simulations are performed for a particular trajectory tracking task of the 7-DOF space manipulator, of which MLCC is evaluated quantitatively. By in-depth research of the simulation results, significant gap between the values of MLCC when using different initial configurations is analyzed, and the discontinuity of allowable load-carrying capacity is illustrated. The proposed analytical method can be taken as theoretical foundation of feasibility analysis, trajectory optimization, and optimal control of trajectory tracking task in on-orbit load-carrying operations.
\end{abstract}

\section{Introduction}

Space manipulators are playing increasingly important roles in space exploration. In particular for load-carrying operations of large structures during maintenance of satellites [1] and construction of space station [2], using space manipulators to replace astronauts can improve economy and security of on-orbit operations. Due to the great mass and inertia tensor of these objects, it could not only challenge driving capability of joints but also result in instability of the spacecraft base under free-floating condition. Therefore, comprehensive, reasonable, and accurate analysis of loadcarrying capacity for FFSM is very necessary. As the basis of various on-orbit operations [3], continuous trajectory tracking task is considered in this paper.

Most of the previous works on load-carrying problems are carried out for FFSM. Aghili and Namvar [4] and Wang et al. [5], respectively, adopted impedance control and adaptive sliding mode control method to improve the reliability and stability during load-carrying process. In order to deal with the trajectory tracking and vibration suppression of a rigid-flexible coupling FFSM with a rigid payload, the composite control approach which combines a nonsingular terminal sliding mode control was proposed [6]. Jia et al. [7] and Liu et al. [8] presented trajectory planning methodologies to achieve the goal of payload maximization, in which optimal joint trajectories were obtained to improve load-carrying capacity of FFSM in point-to-point motion. However, these studies only focused on optimization of dynamic performance for space manipulators and lacked intuitive and systematic analysis of load-carrying capacity.

Load-carrying capacity always depends on the dynamic motion or trajectory of the end-effector [9]. The minimum value of dynamic load-carrying capacity (DLCC) shows the maximum allowable payload that the manipulator can repeatedly lift, which is defined as maximum loadcarrying capacity (MLCC) [10]. In order to evaluate MLCC of manipulators along the given trajectory of end-effector, separate computation of the joint torques for compensating the payload dynamics $\tau_{l}$ and the manipulator dynamics $\tau_{n l}$ is adopted by Wang and Ravani [11]. Crane and Duffy [12] substituted mass of the payload as unknown variables 
into the dynamic equations which are established using Kane method and then calculated MLCC using least square method. The studies mentioned above can only be applied to fixed-base manipulator, whose motion is identical under no-load and load condition, but is not fit for nonholonomic manipulators. To solve this problem, Korayem and Ghariblu [13] established augmented Jacobi matrix of ground wheeled robot using nonholonomic constraint and redundancy additional constraints and then evaluated MLCC through similar description method of [11] with taking into account kinematic singularity problem. In contrast, the generalized Jacobi matrix of FFSM contains the dynamic parameters, so that dynamic singularity is needed to be considered [14]. Besides that, different from rolling contact between wheels and ground, position and orientation of the spacecraft base are both uncontrolled, which causes high nonlinearity of dynamic equations. Consequently, MLCC of FFSM needs to be achieved through numerical iteration. Using numerical techniques, Korayem et al. [15, 16] also analyzed loadcarrying capacity as optimal control problem, in which a two-point boundary value problem (TPBVP) was established to determine the maximum payload and the corresponding joint optimal path to track a given end-effector trajectory. This method simplified the payload as a mass point but ignored the influence to MLCC caused by inertia tensor of the payload, which is also unfit for FFSM.

It is worth noting that the studies mentioned above (see $[11-13,15,16])$ are all carried out for ground manipulators, in which only joint driving capacity is considered as the primary limitation of MLCC. Furthermore, motion of the manipulator can result in translation and rotation of the base under microgravity condition, which depend on the mass and inertia tensor of the payload. Different from tip over stability problem of ground mobile manipulators caused by gravity [17], base attitude disturbance could have a great influence on communication system and solar panels of spacecraft, which must be considered in load-carrying capacity analysis of FFSM. In addition to this, most previous studies only evaluated MLCC for the given trajectory of endeffector and corresponding joint path. In trajectory tracking task of FFSM, pose of the end-effector is not only related to the current joint angles but also associated with the history of joint movements, which causes two problems when linear velocity and angular velocity of the end-effector are determined. On the one hand, generalized Jacobi matrix of FFSM is different when the payload changes, which may result in singularity of the manipulator and instability of the base due to the variation of joint path and base attitude disturbance. FFSM will be unable to carry certain object and move along the trajectory even if mass and inertia tensor of the body are not greater than the "maximum payload." On the other hand, for redundant space manipulators, there may be multiple initial configurations for the starting point of a given trajectory tracking task, whose corresponding joint path solved by velocity-level inverse kinematic equations can make a massive difference of DLCC. Therefore, in order to comprehensively analyze the load-carrying capacity of FFSM in trajectory tracking task, the mentioned two cases must be considered.
This paper is organized as follows. Section 2 derives the kinematic and dynamic equations of FFSM with a payload; Section 3 formulates evaluation of DLCC as a multiconstrained nonlinear programming problem, and an efficient algorithm is presented to determine maximum payload within discontinuous feasible region. In Section 4, the maximum payload initial configuration analysis method based on PSO is explained. Section 5 shows the simulation results and analyzes load-carrying capacity of 7-DOF FFSM in typical trajectory tracking task. Section 6 presents the conclusions of the work.

\section{Mathematical Model of FFSM with a Payload}

As shown in Figure 1, the system considered in this paper consists of a base, a revolute-jointed manipulator which has $n$ degrees of freedom, and a payload which is attached to the end-effector. It is assumed that the components of the system are all rigid bodies.

Define the symbols as follows:

$\Sigma_{I}$ : inertial coordinate system, which is the reference coordinate system of all recursive calculations,

$\Sigma_{b}$ : coordinate system of the base,

$\Sigma_{k}$ : coordinate system of link $k$,

$\Sigma_{E}$ : coordinate system of the end-effector,

$J_{k}$ : joint $k$ of the manipulator, which is used to connect link $k-1$ with link $k$,

$C_{k}$ : mass center of link $k$,

$\mathbf{p}_{e}$ : vector from $\Sigma_{n}$ to $\Sigma_{E}$,

$m_{k}$ : mass of link $k$,

$\mathbf{I}_{k}$ : inertia tensor of link $k$ with respect to $\Sigma_{k}$.

According to reference [18], the general kinematic equation of space manipulators can be written as

$$
\dot{\mathbf{x}}_{e}=\mathbf{J}_{b} \dot{\mathbf{x}}_{b}+\mathbf{J}_{m} \dot{\mathbf{q}}
$$

where $\dot{\mathbf{x}}_{e}=\left[\mathbf{v}_{e}^{T}, \boldsymbol{\omega}_{e}^{T}\right]^{T} \in \mathbf{R}^{6}$ is velocity vector of the endeffector with respect to $\Sigma_{I} ; \dot{\mathbf{x}}_{b}=\left[\mathbf{v}_{b}^{T}, \boldsymbol{\omega}_{b}^{T}\right]^{T} \in \mathbf{R}^{6}$ is velocity vector of the base with respect to $\Sigma_{I} ; \dot{\mathbf{q}}=\left[\dot{q}_{n}, \dot{q}_{n-1}, \ldots, \dot{q}_{1}\right]^{T} \in$ $\mathbf{R}^{n}$ is joint angular velocity vector; $\mathbf{J}_{b} \in \mathbf{R}^{6 \times 6}$ is Jacobian matrix denoting the relationship between velocity of the base and velocity of the end-effector; $\mathbf{J}_{m} \in \mathbf{R}^{6 \times 6}$ is Jacobian matrix denoting the relationship between joint angular velocity and velocity of the end-effector.

It is assumed that the initial linear and angular momentums are equal to zero and no external forces or torques act on the whole system under free-floating condition. According to conservation of momentum and angular momentum, we can easily obtain that

$$
\mathbf{H}_{b} \dot{\mathbf{x}}_{b}+\mathbf{H}_{b m} \dot{\mathbf{q}}=0
$$




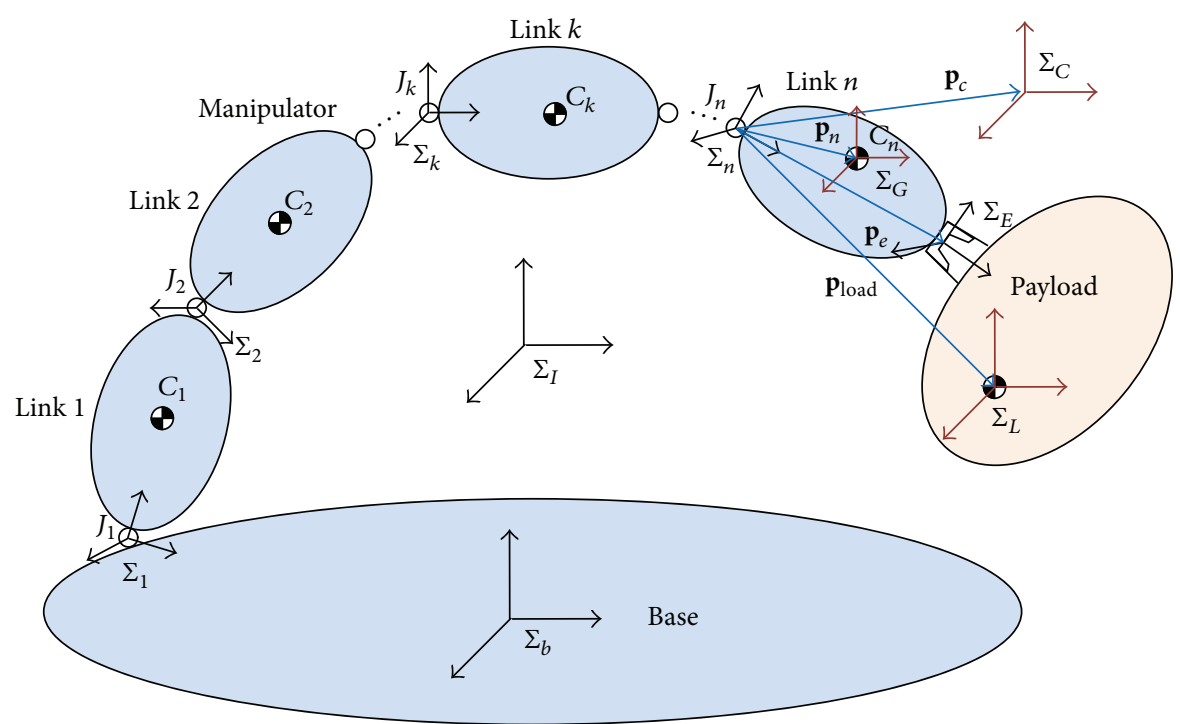

FIGURE 1: Simplified model of free-floating space manipulators.

where $\mathbf{H}_{b}$ and $\mathbf{H}_{b m}$ denote inertia matrix of base and coupled inertia matrix, respectively [18]. Substituting (2) into (1) leads to the relationship as follows:

$$
\begin{aligned}
\dot{\mathbf{x}}_{e} & =\mathbf{J}_{\text {float }} \dot{\mathbf{q}} \\
\dot{\mathbf{x}}_{b} & =\mathbf{J}_{b m} \dot{\mathbf{q}},
\end{aligned}
$$

where $\mathbf{J}_{\text {float }}=\mathbf{J}_{m}-\mathbf{J}_{b} \mathbf{H}_{b}^{-1} \mathbf{H}_{b m}$ denotes the generalized Jacobian matrix of space manipulators; $\mathbf{J}_{b m}=-\mathbf{H}_{b}^{-1} \mathbf{H}_{b m}$ denotes the relationship between joint angular velocity and base velocity.

Link $n$ and payload can be treated as a single composite rigid body and $\Sigma_{G}$ and $\Sigma_{L}$ are defined as coordinate system at the mass center of link $n$ and payload, respectively, while $\Sigma_{C}$ is defined as coordinate system at the mass center of the composite rigid body. Using parallel-axis theorem and rotation transformation of the inertia tensor, mass and inertia tensor of the composite body can be easily obtained as

$$
\begin{gathered}
m_{c}=m_{n}+m_{\text {load }} \\
m_{c} \mathbf{p}_{c}=m_{n} \mathbf{p}_{n}+m_{\text {load }} \mathbf{p}_{\text {load }} \\
\mathbf{I}_{c}={ }^{C} \mathbf{I}_{n}+{ }^{C} \mathbf{I}_{\text {load }} \\
{ }^{C} \mathbf{I}_{n}={ }^{C} \mathbf{R}_{G}\left[\mathbf{I}_{n}+m_{n}\left({ }^{C} \mathbf{P}_{G}^{T}{ }^{C} \mathbf{P}_{G} \mathbf{E}_{3}-{ }^{C} \mathbf{P}_{G}{ }^{C} \mathbf{P}_{G}^{T}\right)\right]{ }^{C} \mathbf{R}_{G}^{T} \\
{ }^{C} \mathbf{I}_{\text {load }}={ }^{C} \mathbf{R}_{L}\left[\mathbf{I}_{\text {load }}+m_{\text {load }}\left({ }^{C} \mathbf{P}_{L}{ }^{T} \mathbf{P}_{L} \mathbf{E}_{3}-{ }^{C} \mathbf{P}_{L}{ }^{C} \mathbf{P}_{L}^{T}\right)\right]{ }^{C} \mathbf{R}_{L}^{T},
\end{gathered}
$$

where $m_{n}, m_{\text {load }}$, and $m_{c}$ denote mass of link $n$, payload, and the composite body, respectively; $\mathbf{p}_{n}$ denotes vector from $\Sigma_{n}$ to $\Sigma_{G}, \mathbf{p}_{\text {load }}$ and $\mathbf{p}_{c}$ denote vectors from $\Sigma_{n}$ to $\Sigma_{L}$ and $\Sigma_{C}$, respectively; $\mathbf{I}_{n}, \mathbf{I}_{\text {load }}$, and $\mathbf{I}_{c}$ denote inertia tensors of link $n$, payload, and the composite body with respect to their own coordinate system, respectively; ${ }^{C} \mathbf{P}_{G}$ and ${ }^{C} \mathbf{P}_{L}$ denote vectors from $\Sigma_{G}$ and $\Sigma_{L}$ to $\Sigma_{C} ;{ }^{C} \mathbf{R}_{G}$ and ${ }^{C} \mathbf{R}_{L}$ denote the rotation matrix of $\Sigma_{G}$ and $\Sigma_{L}$ with respect to $\Sigma_{C}$.
The velocity and acceleration of the composite body with respect to $\Sigma_{I}$ can be calculated as follows:

$$
\begin{gathered}
\mathbf{v}_{c}=\mathbf{v}_{e}+\omega_{e} \times\left(\mathbf{p}_{c}-\mathbf{p}_{e}\right) \\
\boldsymbol{\omega}_{c}=\boldsymbol{\omega}_{e} \\
\dot{\mathbf{v}}_{c}=\dot{\mathbf{v}}_{e}+\dot{\boldsymbol{\omega}}_{e} \times\left(\mathbf{p}_{c}-\mathbf{p}_{e}\right)+\omega_{e} \times\left[\boldsymbol{\omega}_{e} \times\left(\mathbf{p}_{c}-\mathbf{p}_{e}\right)\right] \\
\dot{\boldsymbol{\omega}}_{c}=\dot{\boldsymbol{\omega}}_{e} .
\end{gathered}
$$
form as

The first two equations in (6) can be written in matrix

$$
\left[\begin{array}{c}
\mathbf{v}_{c} \\
\boldsymbol{\omega}_{c}
\end{array}\right]=\left[\begin{array}{cc}
\mathbf{E}_{3} & {\left[\boldsymbol{\omega}_{e} \times\left(\mathbf{p}_{c}-\mathbf{p}_{e}\right)\right] \boldsymbol{\omega}_{e}^{+}} \\
0 & \mathbf{E}_{3}
\end{array}\right]\left[\begin{array}{c}
\mathbf{v}_{e} \\
\boldsymbol{\omega}_{e}
\end{array}\right]
$$

Define $\mathbf{J}_{c e}=\left[\begin{array}{c}\mathbf{E}_{3}\left[\boldsymbol{\omega}_{e} \times\left(\mathbf{p}_{c}-\mathbf{p}_{e}\right)\right] \boldsymbol{\omega}_{e}^{+} \\ 0 \\ \mathbf{E}_{3}\end{array}\right]$; then $\dot{\mathbf{x}}_{c}=\mathbf{J}_{c e} \dot{\mathbf{x}}_{e}$, and substituting it into (3) we can obtain

$$
\dot{\mathbf{x}}_{c}=\mathbf{J}_{c e} \mathbf{J}_{\text {float }} \dot{\mathbf{q}} .
$$

According to Newton-Euler equations and D'Alembert's principle, the generalized inertial force acting on the composite body can be written as

$$
\mathbf{F}_{c}=\left[\begin{array}{c}
m_{c} \dot{\mathbf{v}}_{c} \\
\mathbf{I}_{c} \dot{\boldsymbol{\omega}}_{c}+\boldsymbol{\omega}_{c} \times\left(\mathbf{I}_{c} \boldsymbol{\omega}_{c}\right)
\end{array}\right] .
$$

Then the joint torques $\tau=\left[\tau_{1}, \tau_{2}, \ldots, \tau_{n}\right]^{T}$ can be obtained according to recursive Newton Euler dynamic equations.

\section{Evaluation of DLCC in Trajectory Tracking Task}

Using velocity-level inverse kinematic equations, three types of Cartesian continuous trajectory tracking of the endeffector can be attained [19]: (1) continuous pose (including 
position and orientation) tracking; (2) continuous position/orientation tracking without disturbance on the base attitude; (3) continuous position/orientation tracking and adjusting the base attitude at the same time. Considering that the movement of the base can be determined in the second type and third type, continuous pose tracking task is emphatically considered in this paper. In this section, limitations of joint driving capacity and disturbance of the base are emphatically considered in evaluation algorithm, which is also suitable for the other two types of trajectory tracking task.

3.1. Continuous Pose Tracking Planning of the End-Effector. Define $\mathbf{q}_{\text {ini }}$ and $\mathbf{x}_{b \text { ini }}$ as initial configuration and initial pose of the base, respectively. The end-effector of space manipulator is required to carry the payload along the given trajectory in Cartesian space and finally reach the desired pose $\mathbf{x}_{e_{-} \text {des }}$. Define $\Delta t$ as the control cycle of joint controller and discretize the trajectory into $s$ points, $s=\left(t_{f}-t_{0}\right) / \Delta t$.

The initial pose of the end-effector $\mathbf{x}_{e_{\text {ini }}}$ can be obtained according to $\mathbf{q}_{\text {ini }}$. Based on the linear and angular velocity planning of the end-effector, $\dot{\mathbf{x}}_{e i}(i=1,2, \ldots, s)$ can be determined. Then joint velocity can be computed as follows:

$$
\dot{\mathbf{q}}=\mathbf{J}_{\text {float }}^{+}\left(\mathbf{x}_{e i}, \mathbf{q}_{i}, \mathbf{p}_{\text {load }}, m_{\text {load }}, \mathbf{I}_{\text {load }}\right) \dot{\mathbf{x}}_{e},
$$

where $\mathbf{J}_{\text {float }}^{+}$is generalized inverse matrix of $\mathbf{J}_{\text {float }}$ for redundant space manipulators.

The joint velocity of point $i$ is as follows:

$$
\mathbf{q}\left(t_{i+1}\right)=\mathbf{q}\left(t_{i}\right)+\dot{\mathbf{q}}\left(t_{i+1}\right) \Delta t
$$

Then the velocity of the base can be obtained as

$$
\dot{\mathbf{x}}_{b}\left(t_{i}\right)=\mathbf{J}_{b m}\left(\mathbf{x}_{e}\left(t_{i}\right), \mathbf{q}\left(t_{i}\right), \mathbf{p}_{\text {load }}, m_{\text {load }}, \mathbf{I}_{\text {load }}\right) \dot{\mathbf{q}}\left(t_{i}\right) .
$$

The attitude disturbance of the base at point $i+1$ is computed as

$$
\mathbf{x}_{b}\left(t_{i+1}\right)=\mathbf{x}_{b}\left(t_{i}\right)+\dot{\mathbf{x}}_{b}\left(t_{i}\right) \Delta t=\left[\begin{array}{ll}
\mathbf{X}_{b}\left(t_{i}\right) & \boldsymbol{\Phi}_{b}\left(t_{i}\right)
\end{array}\right]^{T},
$$

where $\mathbf{X}_{b}$ and $\boldsymbol{\Phi}_{b}$ denote the position and attitude of the base, respectively; $\boldsymbol{\Phi}_{b}=\left[\alpha_{b}, \beta_{b}, \gamma_{b}\right]^{T}$.

Through circular computations for $s$ loops, the joint path and movement of the base can be obtained.

3.2. Problem Statement of MLCC Evaluation. Define $\mu_{i}$ as the dynamic payload coefficient at discrete point $i$ of the given trajectory, and then dynamic load-carrying capacity of space manipulator can be expressed as [13]

$$
\begin{gathered}
m_{i}^{\text {DLCC }}=\mu_{i}^{\max } m_{\text {load }} \\
\mathbf{I}_{i}^{\text {DLCC }}=\mathbf{c}\left(\mu_{i}^{\max }\right) \mathbf{I}_{\text {load }},
\end{gathered}
$$

where $\mathbf{c} \in \mathbf{R}^{1 \times 3}$ denotes the coefficient vector, which consists of positive rational numbers and related to the mass and its distribution of the payload; $\mu_{i}^{\max }$ is the maximum value of $\mu_{i}$, which is defined as the dynamic load-carrying coefficient.
On this basis, we define the maximum payload coefficient as

$$
\lambda_{\max }=\min \left\{\mu_{1}^{\max }, \mu_{2}^{\max }, \ldots, \mu_{s}^{\max }\right\}
$$

For a given trajectory tracking task, maximum allowable payload that FFSM is able to carry can be defined as MLCC

$$
\begin{gathered}
m_{\mathrm{MLCC}}=\min \left\{m_{i}^{\mathrm{DLCC}}\right\}=\lambda_{\max } m_{\mathrm{load}} \\
\mathbf{I}_{\mathrm{MLCC}}=\min \left\{\mathbf{I}_{i}^{\mathrm{DLCC}}\right\}=\mathbf{c}\left(\lambda_{\max }\right) \mathbf{I}_{\mathrm{load}} .
\end{gathered}
$$

Define $\boldsymbol{\Phi}_{b}^{\max }=\left[\alpha_{b}^{\max }, \beta_{b}^{\max }, \gamma_{b}^{\max }\right]^{T}$ as the maximum allowable attitude disturbance of the base and the constraint function can be expressed as follows:

$$
\begin{aligned}
& g_{1}(\lambda)=\max \left\{\left|\alpha_{b}^{i}(\lambda)\right|\right\}-\alpha_{b}^{\max } \\
& g_{2}(\lambda)=\max \left\{\left|\beta_{b}^{i}(\lambda)\right|\right\}-\beta_{b}^{\max } \\
& g_{3}(\lambda)=\max \left\{\left|\gamma_{b}^{i}(\lambda)\right|\right\}-\gamma_{b}^{\max }
\end{aligned}
$$

Define $\left[\tau_{k}^{\min }, \tau_{k}^{\max }\right]$ as the driving torque range provided by joint $k$; joint drive capacity constraint functions are obtained as

$$
\begin{aligned}
& h_{1}^{k}(\lambda)=\max \left\{\tau_{k}^{i}(\lambda)\right\}-\tau_{k}^{\max } \\
& h_{2}^{k}(\lambda)=\tau_{k}^{\min }-\min \left\{\tau_{k}^{i}(\lambda)\right\} .
\end{aligned}
$$

Considering dynamic parameters of the payload contained within $\mathbf{J}_{\text {float }}$, the joint angle and angular velocity may exceed the limitations when $\lambda$ changes according to (10) and (11). Define $q_{k}^{\max }$ and $q_{k}^{\min }$ as the upper and lower limits of joint $k$, while defining $\dot{q}_{k}^{\max }$ as the upper limit of absolute value of angular velocity for joint $k$; then the corresponding constraint functions are

$$
\begin{aligned}
& h_{3}^{k}(\lambda)=\max \left\{q_{k}^{i}(\lambda)\right\}-q_{k}^{\max } \\
& h_{4}^{k}(\lambda)=q_{k}^{\min }-\min \left\{q_{k}^{i}(\lambda)\right\} \\
& h_{5}^{k}(\lambda)=\max \left\{\left|\dot{q}_{k}^{i}(\lambda)\right|\right\}-\dot{q}_{k}^{\max }
\end{aligned}
$$

In addition, singularity problem of $\mathbf{J}_{\text {float }}$ may happen when using velocity-level inverse kinematic equations as (10). Joint angular velocity would become unacceptably large with singular configurations, which is contained within (19). Considering the multiconstraints mentioned above, evaluation of MLCC can be expressed as a nonlinear programming problem:

$$
\begin{array}{ll}
\max & \lambda \\
\text { s.t. } & g_{j}(\lambda) \leq 0, \quad j=1,2,3 ; \\
& h_{j^{\prime}}^{k}(\lambda) \leq 0, \quad j^{\prime}=1,2, \ldots, 5 ; k=1,2, \ldots, n .
\end{array}
$$


3.3. Solution Algorithm of Maximum Load-Carrying Coefficient. To solve the nonlinear programming problem established above, initial payload needs to be selected. However, feasible region of independent variable in (20) may be discontinuous due to the constraint equations, whose monotonicity is difficult to prove. If line search is executed along the monotone increasing direction of $\lambda$, the solution may be locally optimal, which cannot reflect the true value of MLCC. In order to avoid this problem, maximum payload coefficient can be solved as follows.

(1) Select $m_{0}, \mathbf{I}_{0}$, and $\mathbf{c}(\lambda)$ of the initial payload (according to $m_{\text {load }}$ and $\mathbf{I}_{\text {load }}$ if the object is given), which is heavy enough to dissatisfy all the constraints of (20).

(2) Define $D$ as the number of repetitive line search. Execute searching along monotonic decrease direction of $\lambda$ until reaching the feasible region; $I_{D}$ denotes the number of iterations, and the corresponding result of current line search is marked as $\lambda\left(I_{D}\right)$; the initial region of search is $[0,1]$, and the starting point is $\lambda_{0}=1$.

(3) To ensure computational efficiency, select a proper $\Delta \lambda$ as the initial step size (the obtained $\lambda_{\max }$ may be a lot smaller than actual value if $\Delta \lambda$ is too large, while computational cost would increase if it is too small); for $D=2,3, \ldots$, the corresponding region of line search and step size are $\left[\lambda\left(p_{D-1}\right), \lambda\left(p_{D-1}-1\right)\right]$ and $2^{-(D-1)} \Delta \lambda$, respectively.

(4) In order to reduce the computational cost, compute variables in the following order and determine whether corresponding constraints are satisfied: $\mathrm{J}_{\text {float }} \rightarrow \dot{\mathbf{q}} \rightarrow \mathbf{q} \rightarrow \Phi_{b} \rightarrow \boldsymbol{\tau}$.

(5) In order to get the optimal solution which is close enough to boundary of feasible region, $\lambda\left(p_{D}\right) \in\left\{\mathbf{S}_{1}\right\} \cap$ $\left\{\mathbf{S}_{2}\right\}$ is determined as termination condition of the algorithm. $\left\{\mathbf{S}_{1}\right\}$ is the feasible region of (20), and $\left\{\mathbf{S}_{2}\right\}$ is defined as follows:

$$
\begin{aligned}
\mathbf{S}_{2}=\{\lambda \mid & \left|g_{1}(\lambda)\right| \leq \Delta \alpha_{b} \vee\left|g_{2}(\lambda)\right| \leq \Delta \beta_{b} \\
& \vee\left|g_{3}(\lambda)\right| \leq \Delta \beta_{b} \vee\left|h_{1}^{k}(\lambda)\right| \leq \Delta \tau_{k} \\
& \vee\left|h_{2}^{k}(\lambda)\right| \leq \Delta \tau_{k} \vee\left|h_{3}^{k}(\lambda)\right| \leq \Delta q_{k} \\
& \vee\left|h_{4}^{k}(\lambda)\right| \leq \Delta q_{k} \vee\left|h_{5}^{k}(\lambda)\right| \leq \Delta \dot{q}_{k} ; \\
& i=1,2, \ldots, s ; k=1,2, \ldots, n\},
\end{aligned}
$$

where $\Delta \alpha_{b}, \Delta \beta_{b}, \Delta \gamma_{b}, \Delta \tau_{k}, \Delta q_{k}, \Delta \dot{q}_{k}$ is the accuracy at maximum payload coefficient computation, which denotes the allowable error of constraint functions. When the payload coefficient value lies in feasible region and satisfies (21), it is regarded as the approximate solution of $\lambda_{\max }$.

Using the mentioned algorithm we can efficiently obtain optimal solution of (20) through repeated line search within discontinuous feasible region. When the constraints cannot be satisfied, $\dot{\mathbf{x}}_{e}$ and $\mathbf{q}_{\text {ini }}$ must be reelected. For a given trajectory of the end-effector and corresponding joint path,
MLCC can be evaluated according to Figure 2, which can also be applied in feasibility evaluation of load-carrying capacity when the object is known. If no proper discretized point along the given trajectory can be determined for $\lambda \in[0,1]$, it means space manipulators are incompetent to accomplish the given trajectory tracking task.

\section{Analysis of Maximum Payload Initial Configuration Using PSO}

For redundant space manipulators, there are multiple initial configurations for a given starting point of the task. According to Section 3, MLCC can therefore be significantly different when tracking the same trajectory of end-effector. In order to evaluate the maximum load that the manipulator can carry under all possible circumstances, maximum payload initial configuration planning method is proposed in this section, which provides evidence for further analysis of loadcarrying capacity of the space manipulator.

Firstly, position-level inverse kinematics of redundant space manipulator based on numerical approach is adopted to solve $\mathbf{q}_{\text {ini }}$ which satisfy the given $\mathbf{X}_{e \text {-ini }}$. Define $\mathbf{T}_{\text {ini }}$ as the pose matrix form of $\mathbf{X}_{e-\text { ini }}$ with respect to $\Sigma_{b}$. For a random joint angle vector $\mathbf{q}_{r} \in \mathbf{R}^{n}$, the corresponding pose matrix of the end-effector is defined as $\mathbf{T}_{r}$. It is assumed that the endeffector can move from $\mathbf{T}_{r}$ to $\mathbf{T}_{\text {ini }}$ through differential motion of the joints, and Newton-Raphson iterative equation can be established:

$$
d \mathbf{q}=\mathbf{J}_{m}^{+} \mathbf{D},
$$

where $\mathbf{D}=\left[\begin{array}{llllll}d x & d y & d z & \delta x & \delta y & \delta z\end{array}\right]^{T}$ denotes differential motion vector of the end-effector and $\mathbf{J}_{m}$ denotes motion relationship between the end-effector and the base (assume that it is fixed).

It is assumed that the end-effector moves from current pose to the desired position and orientation through differential movement of the joints, which means

$$
\mathbf{T}_{\text {ini }}=\mathbf{T}_{r}\left(\mathbf{E}_{4}+\Delta\right),
$$

where $\Delta=\operatorname{Trans}(d x, d y, d z) \operatorname{Rot}(k, d \theta)-\mathbf{E}_{4}$ denotes the differential operators. Then the elements of $\mathbf{D}$ can be determined according to $\Delta=\mathbf{T}_{r}^{-1} \mathbf{T}_{\text {ini }}-\mathbf{E}_{4}$.

When the desired accuracy $\xi$ and maximum iterations $\mathbf{N}$ are given, execute iterative computation and save the obtained $\mathbf{q}_{\text {ini }}$ till it satisfies $\|d \mathbf{q}\| \leq \xi$ or output failure information if the accuracy cannot reach the given accuracy when the number of iterations is equal to $\mathbf{N}$.

Consider that the closest joint angles near current configuration can be approached in the iteration process. As a result, the unique feasible initial configuration $\mathbf{q}_{\text {ini }}$ can be obtained according to a given $\mathbf{q}_{r}$, which is regarded as the optimal control vector. Then the maximum payload initial configuration can be solved through optimal computation in $n$-dimensional joint space for the given motion planning of the end-effector. The cost function is expressed as follows by considering $\lambda \geq 0$ :

$$
f=-\lambda_{\max }\left(\mathbf{q}_{r}\right) .
$$




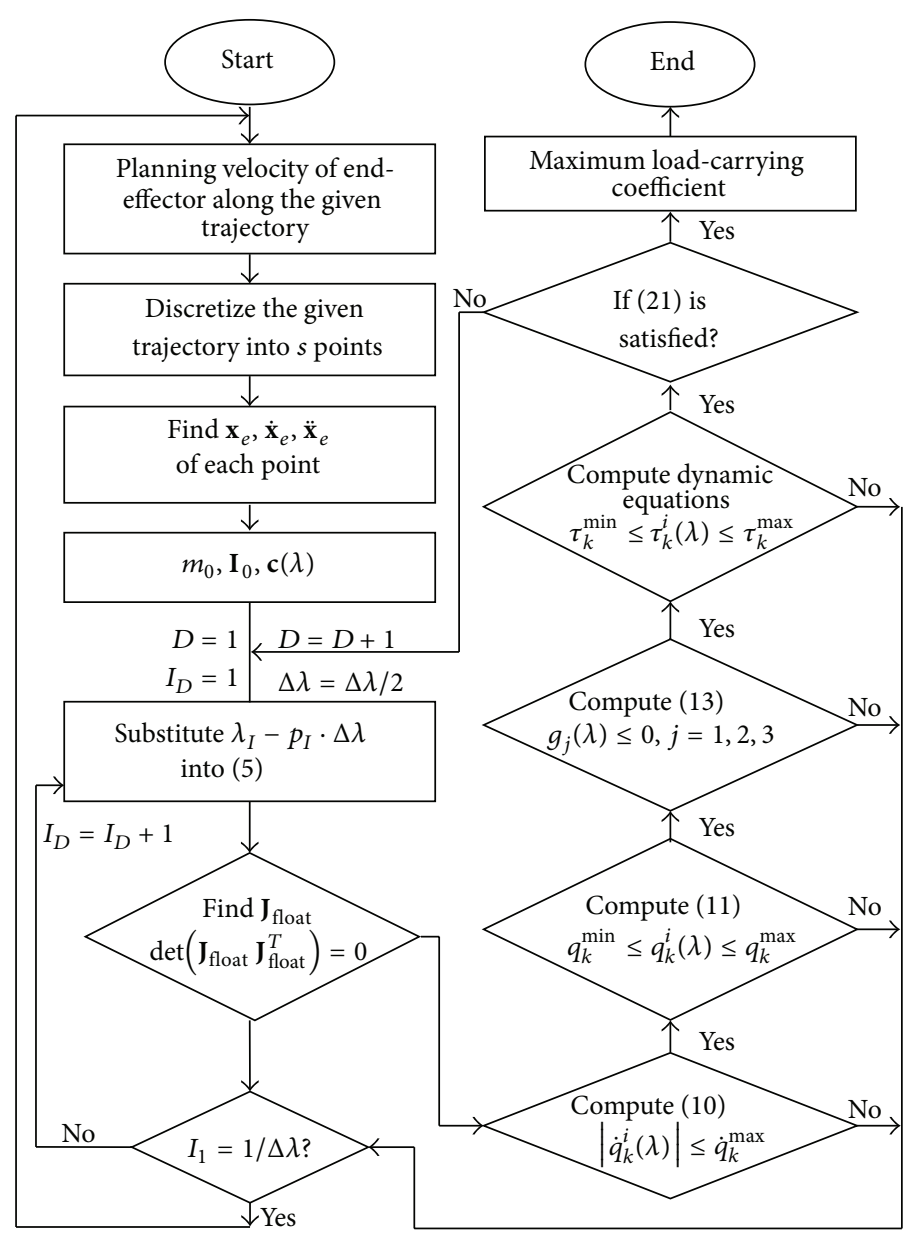

FIGURE 2: Flow chart of maximum payload coefficient solving algorithm.

Particle swarm optimization algorithm is a method that starts from random solutions and searches the global optimal solution through iterative computation, which has advantages of high precision, fast convergence, and so on [20]. When particle [i] of the population evolves to the $k$ th generation within $r$-dimensional continuous searching space, its position and speed can be updated according to the following computation:

$$
\begin{aligned}
& v_{k+1}^{[i]}=\chi v_{k}^{[i]}+c_{1} r_{1}\left(x_{\text {best }}^{[i]}-x_{k}^{[i]}\right)+c_{2} r_{2}\left(g_{\text {best }}-x_{k}^{[i]}\right) \\
& x_{k+1}^{[i]}=x_{k}^{[i]}+v_{k}^{[i]},
\end{aligned}
$$

where $x_{\text {best }}^{[i]}$ denotes the best position that particle [i] experienced; $g_{\text {best }}$ denotes the best position that all the particles experienced; $\chi$ denotes the inertia weight; $c_{1}$ and $c_{2}$ are used to regulate the step size where the particles fly to $x_{\text {best }}^{[i]}$ and $g_{\text {best }}$, respectively; $r_{1}$ and $r_{2}$ are independent random numbers that evenly distribute in $[0,1]$. follows.

In this study, the process for implementing the PSO is as

Step 1. Initialize the population and essential parameters of $\mathrm{PSO}$; the region in each direction of searching space is set as $\left[q_{\min }, q_{\max }\right]$. Then initialize random $\mathbf{q}_{r}$ as initial position of particles and determine initial velocity.

Step 2. Compute the corresponding fitness value of each particle according to the following process.

(a) Solve the corresponding $\mathbf{q}_{\text {ini }}$ of each particle through Newton-Raphson iteration; if the failure information is obtained, then execute (b); else execute (c).

(b) Assign 0 as the fitness value of the particle.

(c) Evaluate MLCC using the algorithm as shown in Figure 2 according to the obtained $\mathbf{q}_{\text {ini }}$. If $\lambda_{\max }=0$, execute (b); else determine the fitness value by substituting the obtained maximum payload coefficient into (24).

Step 3. Compare the current position of each particle with $x_{\text {best }}^{[i]}$ according to its fitness value and then replace $x_{\text {best }}^{[i]}$ with the current position if it is superior to $x_{\text {best }}^{[i]}$. Determine $g_{\text {best }}$ using the fitness values of all the particles and then update the velocity and position of particles according to (25).

Step 4. Loop to Step 2 until the maximum number of generations is not met; else output the corresponding $\mathbf{q}_{\text {ini }}$ of optimal 


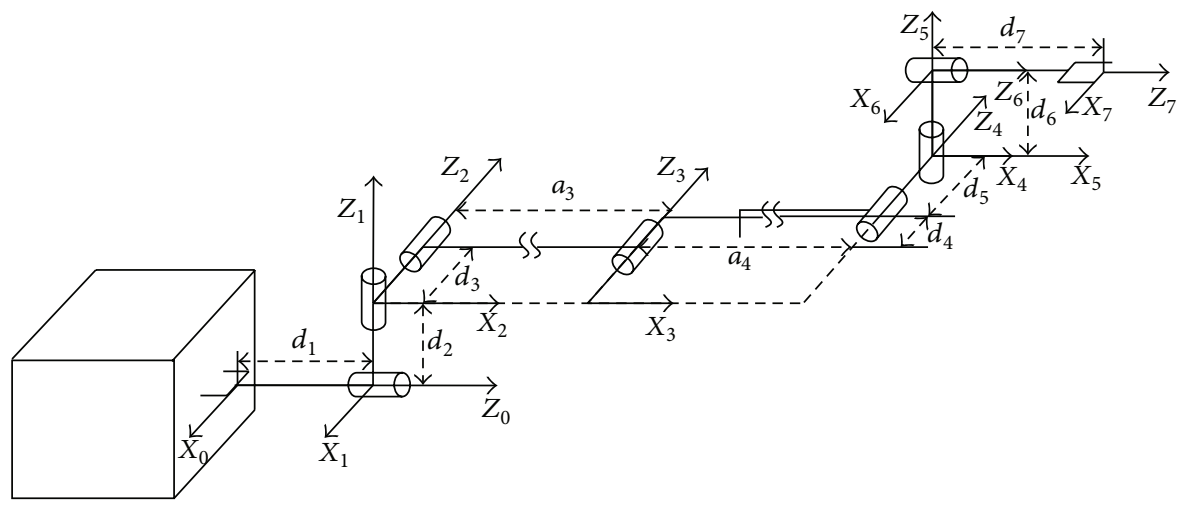

FIGURE 3: 7-DOF free-floating space manipulator.

TABLE 1: $D-H$ parameters of 7-DOF space manipulator.

\begin{tabular}{lcccc}
\hline Link $i$ & $\theta_{i}\left(^{\circ}\right)$ & $d_{i}(\mathrm{~m})$ & $a_{i-1}(\mathrm{~m})$ & $\alpha_{i-1}\left(^{\circ}\right)$ \\
\hline 1 & $\theta_{1}(0)$ & $d_{1}$ & 0 & 90 \\
2 & $\theta_{2}(90)$ & $d_{2}$ & 0 & -90 \\
3 & $\theta_{3}(0)$ & 0 & $a_{3}$ & 0 \\
4 & $\theta_{4}(0)$ & $d_{3}+d_{4}+d_{5}$ & $a_{4}$ & 0 \\
5 & $\theta_{5}(0)$ & 0 & 0 & 90 \\
6 & $\theta_{6}(-90)$ & $d_{6}$ & 0 & -90 \\
7 & $\theta_{7}(0)$ & $d_{7}$ & 0 & 0 \\
\hline
\end{tabular}

solution as the maximum payload initial configuration for the given trajectory tracking task.

\section{Simulation Results}

5.1. Simulation Model. In this case study, a seven-link space manipulator mounted on a base is considered, where $d_{1}=$ $d_{7}=1.2 \mathrm{~m}, d_{2}=d_{3}=d_{5}=d_{6}=0.53 \mathrm{~m}, a_{3}=a_{4}=5.8 \mathrm{~m}$, and $d_{4}=0.52 \mathrm{~m}$. Joint frames according to DH method are shown in Figure 3 and the relative parameters are listed in Tables 1 and 2 . Pose of $\sum_{1}$ is $\left[-0.2 \mathrm{~m}, 0 \mathrm{~m}, 2 \mathrm{~m}, 0^{\circ}, 0^{\circ}, 0^{\circ}\right]$ with respect to $\Sigma_{b}$ as shown in Figure 3.

5.2. Results and Discussions. Simulations are performed to analyze load-carrying capacity of 7-DOF FFSM when tracking a given straight line in Cartesian space. $\mathbf{x}_{b_{-} \text {ini }}=$ $[0,0,0,0,0,0]^{T}$; initial pose of the end-effector is set as $\mathbf{x}_{e_{\text {_ini }}}=[-4.55 \mathrm{~m},-6.7 \mathrm{~m}, 7.85 \mathrm{~m}, 1.5 \mathrm{rad}, 0,-3 \mathrm{rad}]^{T}$, while the desired pose of the end-effector is $\mathbf{x}_{e_{-} \text {des }}=$ $[-2 \mathrm{~m},-7.5 \mathrm{~m}, 4 \mathrm{~m}, 1.6 \mathrm{rad},-0.5 \mathrm{rad},-3 \mathrm{rad}]^{T}$; the angular velocity and linear velocity of the end-effector are determined by conventional trapezoidal-velocity profile [21], in which the total time is set as $80 \mathrm{~s}$ and acceleration and deceleration times are both set as 20 seconds; $\Delta t=100 \mathrm{~ms}$.

Parameters and constraints are set as follows: $m_{0}=3 e+$ $04 \mathrm{~kg}, I_{x x}=I_{z z}=1.08 e+06 \mathrm{~kg} \cdot \mathrm{m}^{2}, I_{y y}=7.6 e+04 \mathrm{~kg} \cdot \mathrm{m}^{2}$, and $\mathbf{c}=\left[0.035 \lambda+0.956 \lambda^{3}, \lambda, 0.035 \lambda+0.956 \lambda^{3}\right]^{T} ;$ it is assumed that the mass center of payload is a constant $\mathbf{p}_{\text {load }}=[0$, $1.725 \mathrm{~m}, 0]^{T}, \Delta \lambda=0.02$; the range of joint motion is from

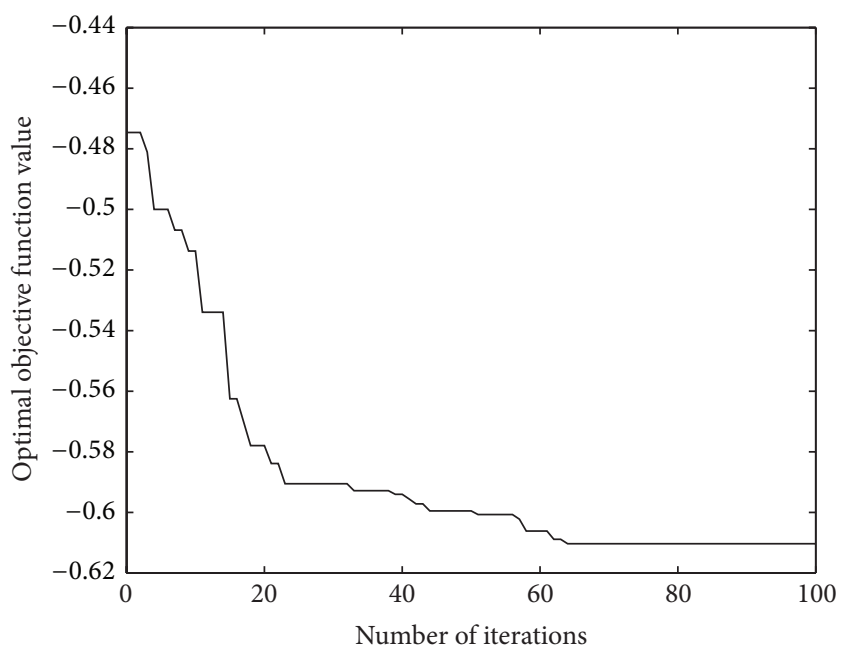

FIGURE 4: Changes of best value in the first case.

$-180^{\circ}$ to $180^{\circ}, \Delta q_{k}=5^{\circ}$; the absolute value of maximum joint angular velocity is $1^{\circ} / \mathrm{s}, \Delta \dot{q}_{k}=0.1^{\circ} / \mathrm{s}$; the limit of joint torques is from $-500 \mathrm{~N} \cdot \mathrm{m}$ to $500 \mathrm{~N} \cdot \mathrm{m}, \Delta \tau_{k}=5 \mathrm{~N} \cdot \mathrm{m}$; maximum allowable attitude disturbance of the base is set as $\left[5^{\circ}, 5^{\circ}\right.$, $5^{\circ}$ ], $\Delta \alpha_{b}=\Delta \beta_{b}=\Delta \gamma_{b}=0.02^{\circ}$; the accuracy of inverse kinematics is $\xi=0.001, \mathbf{N}=100$; the number of particles is $100, c_{1}=c_{2}=2$; the maximum number of iterations is 100 .

Solve maximum payload initial configuration using the algorithm proposed in Section 4. The optimal solution is $[-1.0210,2.7640,-0.5205,1.9840,1.9154,-0.8771,2.6001]$ and the corresponding initial configuration (marked as A) is $\left[-43.64^{\circ},-30.52^{\circ},-8.78^{\circ},-80.62^{\circ}, 95.05^{\circ},-155.86^{\circ}, 53.02^{\circ}\right]$, $f_{\mathrm{A}}=-0.6164$. Contrastive simulations are performed using $f=\lambda_{\max }$ as the objective function. The resulting initial configuration (marked as B) is $\left[-16.04^{\circ}, 5.63^{\circ},-84.71^{\circ}\right.$, $\left.66.31^{\circ},-159.87^{\circ}, 13.56^{\circ},-102.28^{\circ}\right], f_{\mathrm{B}}=0.0753$. Using the two extreme configurations $\mathrm{A}$ and $\mathrm{B}$, the 7-DOF FFSM can possess the maximum and minimum MLCC for the given task. The optimal objective function values of particles varying with the number of iterations in the two experiments are shown in Figures 4 and 5. Let space manipulator carry respective "maximum payload" along the given trajectory: 
TABLE 2: Dynamic parameters of 7-DOF space manipulator.

\begin{tabular}{|c|c|c|c|c|c|c|c|c|}
\hline Parameters & Base & Link 1 & Link 2 & Link 3 & Link 4 & Link 5 & Link 6 & Link 7 \\
\hline \multirow[t]{2}{*}{ Mass/(kg) } & $7.5 e+04$ & 30 & 30 & 70 & 75 & 30 & 30 & 40 \\
\hline & 0 & 0 & -0.265 & 2.9 & 2.7 & 0 & 0 & 0 \\
\hline \multirow[t]{2}{*}{${ }^{i} \mathbf{p}_{i} /(\mathrm{m})$} & 0 & -0.265 & 0 & 0 & 0 & 0 & 0 & 0 \\
\hline & 0 & 0 & 0 & 0 & 0.5 & 0.265 & 0.265 & 0.6 \\
\hline \multicolumn{9}{|l|}{$\mathbf{I}_{k} /\left(\mathrm{kg} \cdot \mathrm{m}^{2}\right)$} \\
\hline$I_{x x}$ & $7.5 e+04$ & 0.98 & 0.57 & 1.32 & 1.91 & 0.98 & 0.98 & 5.18 \\
\hline$I_{y y}$ & $7.5 e+04$ & 0.57 & 0.98 & 197.2 & 243.4 & 0.98 & 0.98 & 5.18 \\
\hline$I_{z z}$ & $7.5 e+04$ & 0.98 & 0.98 & 197.2 & 242.9 & 0.57 & 0.57 & 0.75 \\
\hline$I_{x y}$ & 0 & 0 & 0 & 0 & 0 & 0 & 0 & 0 \\
\hline$I_{y z}$ & 0 & 0 & 0 & 0 & 0 & 0 & 0 & 0 \\
\hline$I_{z x}$ & 0 & 0 & 0 & 0 & -4 & 0 & 0 & 0 \\
\hline
\end{tabular}

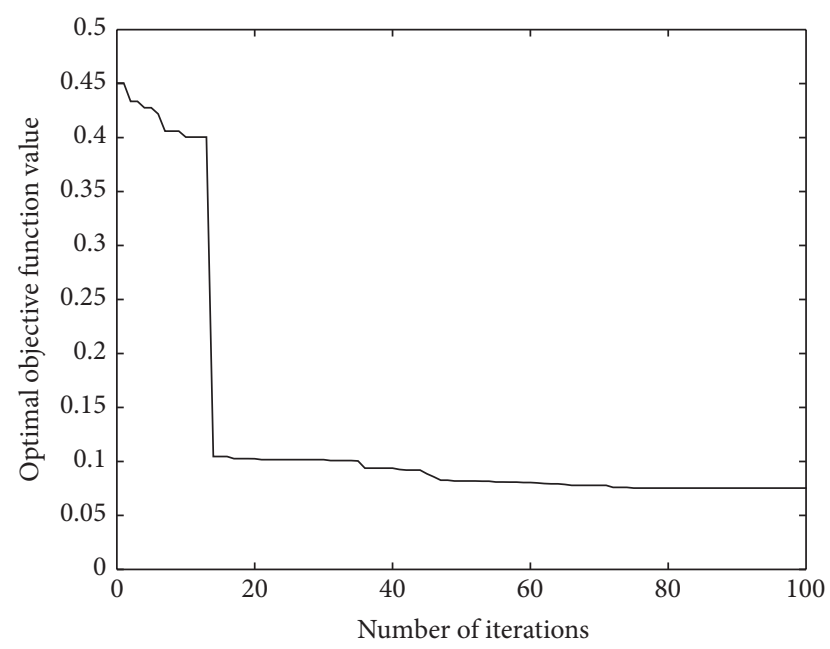

Figure 5: Changes of best value in the second case.

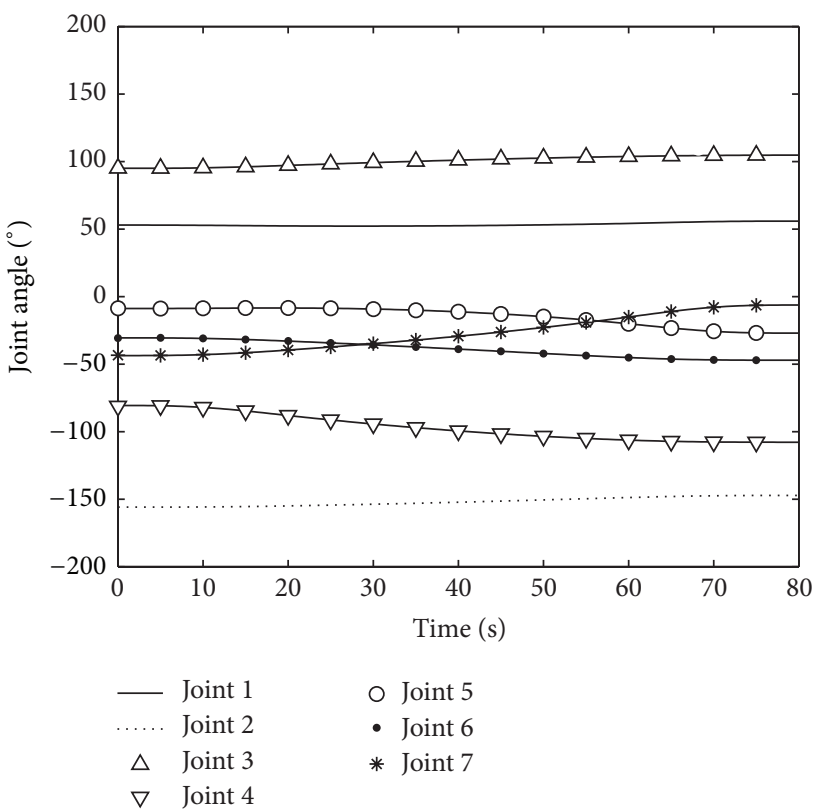

Figure 6: The angle trajectories using A.

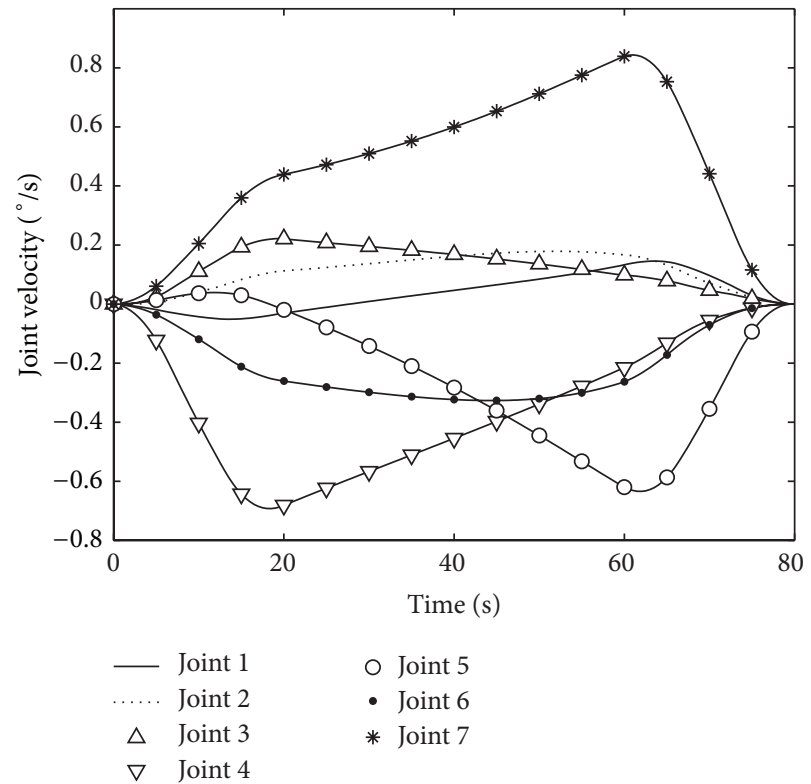

Figure 7: The angle velocity trajectories using A.

when using $\mathrm{A}$ as the initial configuration, joint angle and angular velocity changes are shown in Figures 6 and 7, and the joint torque and base attitude curves are shown in Figures 8(a) and 9(a); when using B as the initial configuration, the joint torque and base attitude curves are shown in Figures 8(b) and 9(b). In the first case, maximum payload coefficient is $\lambda_{\text {max }}^{\mathrm{A}}=0.6103$, which means that the manipulator can carry the payload with a great mass of $18.31 \mathrm{t}$, while, in the second case, $\lambda_{\max }^{\mathrm{B}}=0.0753$, which means that the mass of maximum payload cannot be larger than $2.26 \mathrm{t}$. Thus, for a given trajectory tracking task of redundant space manipulator, an appropriate initial configuration can significantly improve MLCC of free-floating space manipulator.

Further simulations are performed to illustrate the reason for significant differences of MLCC when using A and B. Define $\phi_{b}^{\max }=\max \left\{\alpha_{b}^{\max }, \beta_{b}^{\max }, \gamma_{b}^{\max }\right\}$ and $\tau_{\max }=\max \left\{\left|\tau_{k}^{\min }\right|\right.$, $\left.\left|\tau_{k}^{\max }\right|\right\}$; then 400 different initial configurations are solved by using Newton-Raphson iterative method. Using the 400 sets 


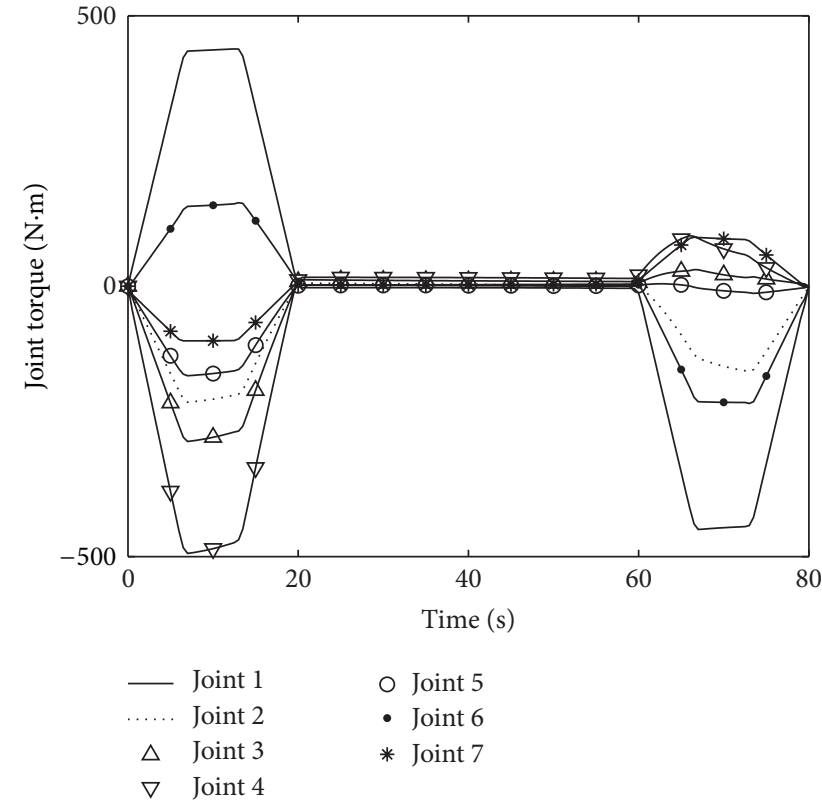

(a) Using initial configuration $\mathrm{A}$

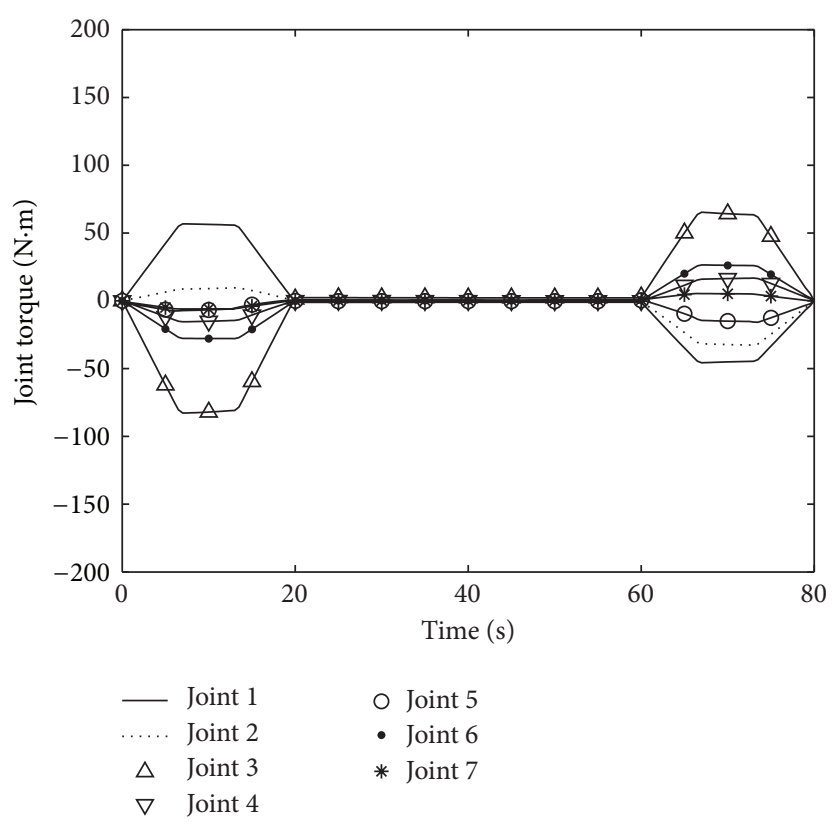

(b) Using initial configuration B

FIgURE 8: Torques of joints under maximum payload condition.

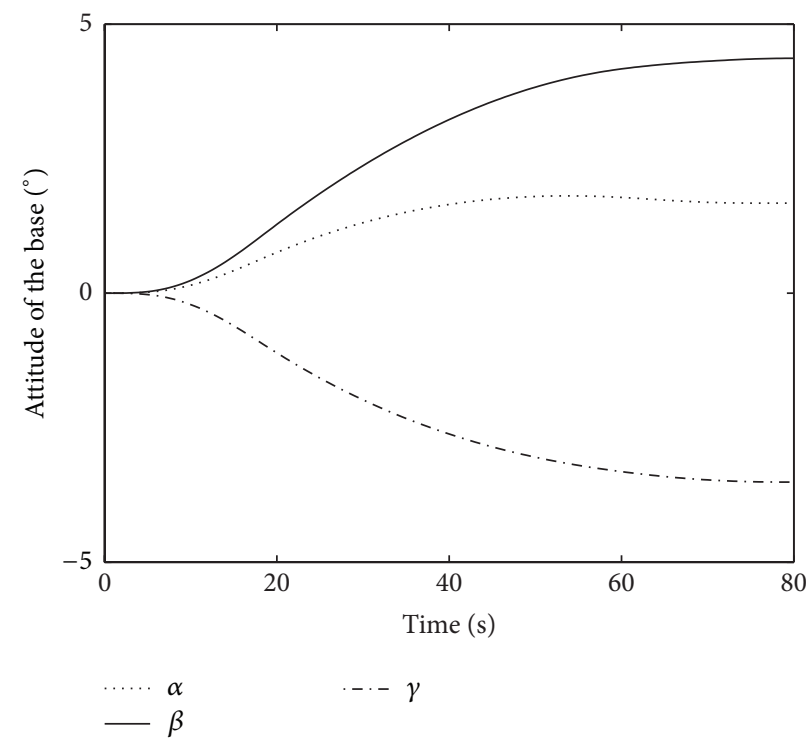

(a) Initial configuration A

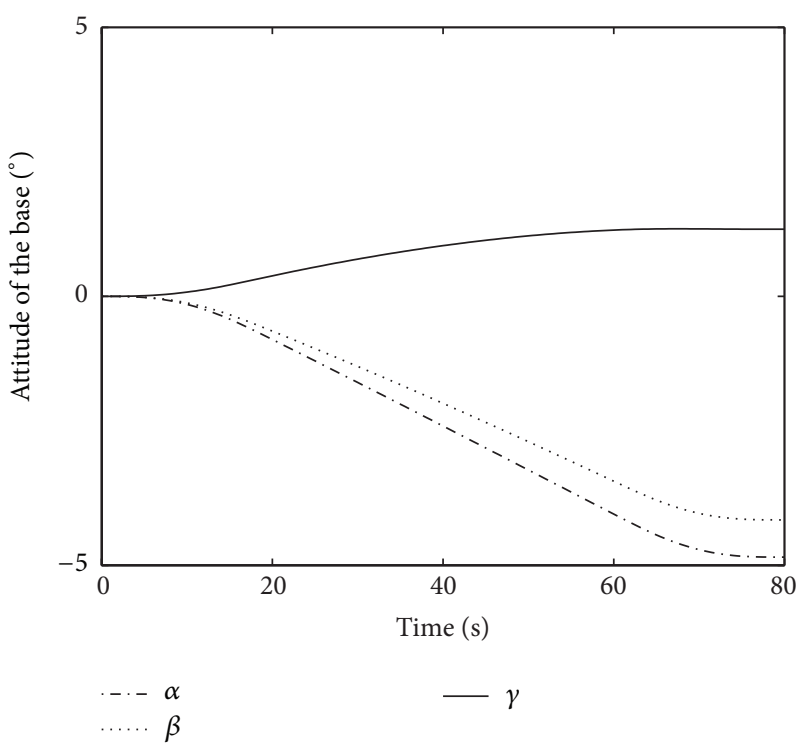

(b) Initial configuration $B$

FIGURE 9: Attitude disturbance of the base under maximum payload condition.

of initial configurations to carry a payload along the given trajectory, $\phi_{b}^{\max }$ and $\tau_{\max }$ with respect to payload coefficient are obtained as shown in Figures 10 and 11, respectively. According to Figures 8-11, when using A to carry the "maximum payload", $\tau_{\max }^{\mathrm{A}}=499.43 \mathrm{~N} \cdot \mathrm{m} \in[464.03,500]$ reaches the boundary of feasible region while $\phi_{b}^{\max }=4.77^{\circ} \in\left[4.64^{\circ}, 5^{\circ}\right]$, $\lambda_{\text {max }}^{\mathrm{A}}<0.6164<0.6381$; when using $\mathrm{B}, \phi_{b}^{\max }=4.98^{\circ}$ reaches the boundary of feasible region firstly. On the premise of satisfying joint angle and angular velocity constraints, $\tau_{\max }$ presents a monotonically increasing trend as a whole when $\lambda \in[0,1]$, while $\phi_{b}^{\max }$ is locally monotonic and thus leads to such a significant difference (about a multiple of about 7.1) of MLCC when using with the two initial configurations.

In addition, the maximum payload coefficients when using the 400 different initial configuration do not exceed the following ranges: $[0.0753,0.1098] \cup[0.3882,0.4543] \cup$ $[0.5895,0.6103]$. The range of allowable payload of space manipulator in the given trajectory tracking task can be 


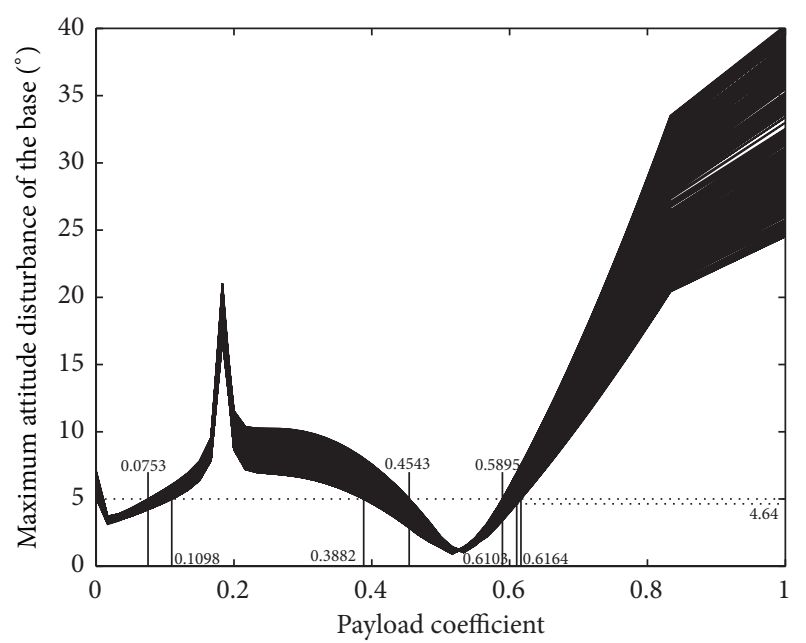

FIGURE 10: $\phi_{b}^{\max }$ with respect to payload coefficient.

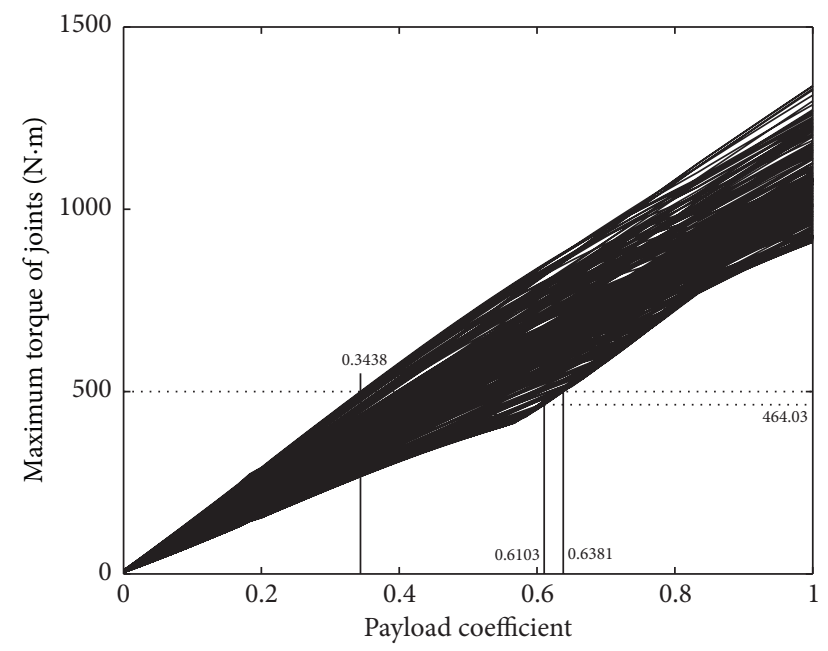

FIGURE 11: $\tau_{\max }$ with respect to payload coefficient.

approximately regarded as $\lambda \in[0,0.1098] \cup[0.3882,0.6103]$. It is scarcely possible to find an appropriate initial configuration for the payload within $\lambda \in[0,0.1098] \cup[0.3882,0.6103]$, which is consistent with the situation that the objective function value suffers a sudden drop from 0.4005 to 0.1045 at the 15th iteration in Figure 5.

When space manipulator carries the "maximum payload" using A, it is assumed that mass of the payload is constant, while multiplying inertia tensor of the payload by a certain proportionality coefficient which varies in $[0.1,1] . \phi_{b}^{\max }$ and $\tau_{\max }$ changing with respect to the proportionality coefficient are shown in Figures 12 and 13. When the proportionality coefficient is about 0.05 and $0.2, \phi_{b}^{\max }$ is $116^{\circ}$ and $50^{\circ}$ while the magnitude of joint torque reaches $10^{8}(\mathrm{~N} \cdot \mathrm{m})$ while attitude disturbance $10^{6}\left(^{\circ}\right)$, which indicates the occurrence of dynamics singularity and base instability condition. Despite excluding these extreme cases, space manipulator cannot be able to accomplish the given task when proportionality coefficient reduces, which proves the incontinuity of feasible

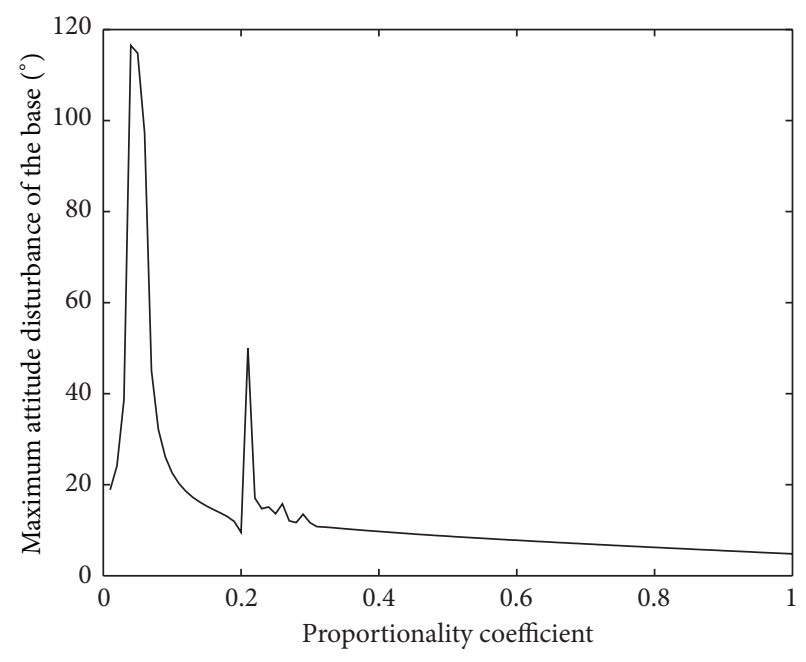

FIGURE 12: $\phi_{b}^{\max }$ with respect to proportionality coefficient.

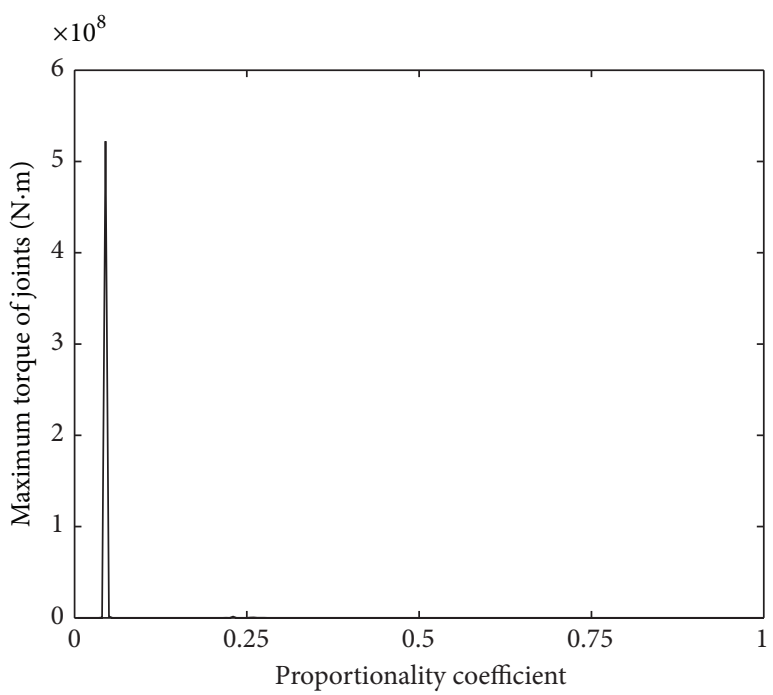

FIGURE 13: $\tau_{\max }$ with respect to proportionality coefficient.

region in (20). All in all, different from the ground fixedbase manipulators which are able to carry arbitrary payload as long as the mass and inertia tensor are not greater than the "maximum load" [11], load-carrying capacity for FFSM has the characteristics of discontinuity.

According to the simulation results and discussions above, we can draw the following conclusions. (1) Driving capability of the joints and attitude disturbance limit of the base are important constraints of MLCC for space manipulators, which must be considered in trajectory planning and control process. (2) Monotonicity of constraint functions results in discontinuous of load-carrying capacity for FFSM, which is essentially caused by nonholonomic characteristic of space manipulators. (3) MLCC of FFSM that only reflects "maximum payload" of the manipulator can repeatedly carry along the given trajectory when achieving upper limit of its ability, which needs to be comprehensively evaluated and 
analyzed with considering allowable range of load-carrying capacity for specific tasks and objects.

\section{Conclusion}

In this paper, a scheme is developed to analyze load-carrying capacity of redundant space manipulators in trajectory tracking task. In the proposed scheme, evaluation of MLCC is described as multiconstrained nonlinear programming problem, which is efficiently solved through repeated line search within discontinuous feasible region. The MLCC can be obtained for a given trajectory and corresponding joint path. After that, considering the influence of MLCC caused by different initial configurations of the manipulator for a given starting point of trajectory, a kind of maximum payload initial configuration planning method is proposed by using PSO algorithm. The simulations comprehensively verify the characteristic of load-carrying capacity for a 7-DOF space manipulator, which also demonstrate the effectiveness of the proposed algorithms. The proposed analytical method provides theoretical foundations of feasibility analysis, trajectory optimizing, and control strategy research of space manipulators in on-orbit load-carrying operations.

\section{Conflict of Interests}

The authors declare that there is no conflict of interests regarding the publication of this paper.

\section{Acknowledgments}

This project is supported by the National Natural Science Foundation of China (61175080), the National Key Basic Research Program of China (2013CB733000), and the Specialized Research Fund for the Doctoral Program of Higher Education (20130005110009).

\section{References}

[1] W.-K. Yoon, T. Goshozono, H. Kawabe et al., "Model-based space robot teleoperation of ETS-VII manipulator," IEEE Transactions on Robotics and Automation, vol. 20, no. 3, pp. 602-612, 2004.

[2] E. Coleshill, L. Oshinowo, R. Rembala, B. Bina, D. Rey, and S. Sindelar, "Dextre: improving maintenance operations on the International Space Station," Acta Astronautica, vol. 64, no. 9-10, pp. 869-874, 2009.

[3] W.-F. Xu, W.-Y. Qiang, C. Li, B. Liang, and Y. Liu, "Progress in path planning of free-floating space robot," Journal of Harbin Institute of Technology, vol. 41, no. 11, pp. 1-12, 2009.

[4] F. Aghili and M. Namvar, "Scaling inertia properties of a manipulator payload for 0-g emulation of spacecraft," International Journal of Robotics Research, vol. 28, no. 7, pp. 883-894, 2009.

[5] C. Wang, X. Pei, and P. Wu, "Composite control of on-orbit operation of free-floating space flexible manipulator with rigid load," Journal of Nanjing University of Aeronautics and Astronautics, vol. 44, no. 4, pp. 452-457, 2012.
[6] C. Wang, P. Wu, X. Zhou, and X. Pei, “Composite sliding mode control for a free-floating space rigid-flexible coupling manipulator system," International Journal of Advanced Robotic Systems, vol. 10, 2013

[7] Q. Jia, Y. Liu, G. Chen, and H. Sun, "Maximum load path planning for space manipulator in point-to-point task," in Proceedings of the IEEE 8th Conference on Industrial Electronics and Applications (ICIEA '13), pp. 988-993, Melbourne, Australia, June 2013.

[8] Y. Liu, Q. Jia, G. Chen, and H. Sun, "Load maximization trajectory optimization for free-floating space robot using multiobjective particle swarm optimization algorithm," Robot, vol. 36, no. 4, pp. 402-410, 2014.

[9] M. H. Korayem and H. Tourajizadeh, "Maximum DLCC of spatial cable robot for a predefined trajectory within the workspace using closed loop optimal control approach," Journal of Intelligent \& Robotic Systems, vol. 63, no. 1, pp. 75-99, 2011.

[10] Y. L. Yao, M. H. Korayem, and A. Basu, "Maximum allowable load of flexible manipulators for given dynamic trajectory," Robotics and Computer Integrated Manufacturing, vol. 10, no. 4, pp. 301-309, 1993.

[11] L. T. Wang and B. Ravani, "Dynamic load carrying capacity of mechanical manipulators-part I: problem formulation," Journal of Dynamic Systems, Measurement and Control, vol. 110, no. 1, pp. 46-52, 1988.

[12] C. D. Crane III and J. Duffy, "A dynamic analysis of a spatial manipulator to determine payload weight," Journal of Robotic Systems, vol. 20, no. 7, pp. 355-371, 2003.

[13] M. H. Korayem and H. Ghariblu, "Maximum allowable load on wheeled mobile manipulators imposing redundancy constraints," Robotics and Autonomous Systems, vol. 44, no. 2, pp. 151-159, 2003.

[14] G. Chen, L. Zhang, Q. Jia, and H. Sun, "Singularity analysis of redundant space robot with the structure of Canadarm2," Mathematical Problems in Engineering, vol. 2014, Article ID 735030, 9 pages, 2014.

[15] H. Korayem and M. Irani, "Maximum dynamic load determination of mobile manipulators via nonlinear optimal feedback," Scientia Iranica Transaction B-Mechanical Engineering, vol. 17, no. 2, pp. 121-135, 2010.

[16] M. H. Korayem and A. Nikoobin, "Maximum payload path planning for redundant manipulator using indirect solution of optimal control problem," International Journal of Advanced Manufacturing Technology, vol. 44, no. 7-8, pp. 725-736, 2009.

[17] M. H. Korayem, V. Azimirad, A. Nikoobin, and Z. Boroujeni, "Maximum load-carrying capacity of autonomous mobile manipulator in an environment with obstacle considering tip over stability," The International Journal of Advanced Manufacturing Technology, vol. 46, no. 5-8, pp. 811-829, 2010.

[18] Y. Xu and H.-Y. Shum, "Dynamic control and coupling of a freeflying space robot system," Journal of Robotic Systems, vol. 11, no. 7, pp. 573-589, 1994.

[19] W.-F. Xu, Y. Liu, W.-Y. Qiang, B. Liang, and C. Li, "Cartesian continuous-path planning for free-floating space robot," Control and Decision, vol. 23, no. 3, pp. 278-287, 2008.

[20] R. Eberhart and J. Kennedy, "New optimizer using particle swarm theory," in Proceedings of the 1995 6th International Symposium on Micro Machine and Human Science, pp. 39-43, October 1995. 
[21] M. Haddad, W. Khalil, and H. E. Lehtihet, "Trajectory planning of unicycle mobile robots with a trapezoidal-velocity constraint," IEEE Transactions on Robotics, vol. 26, no. 5, pp. 954962,2010 


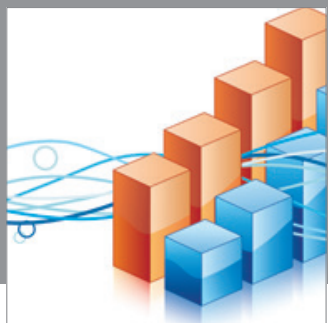

Advances in

Operations Research

mansans

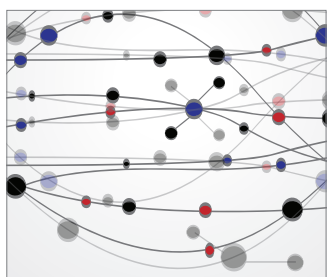

The Scientific World Journal
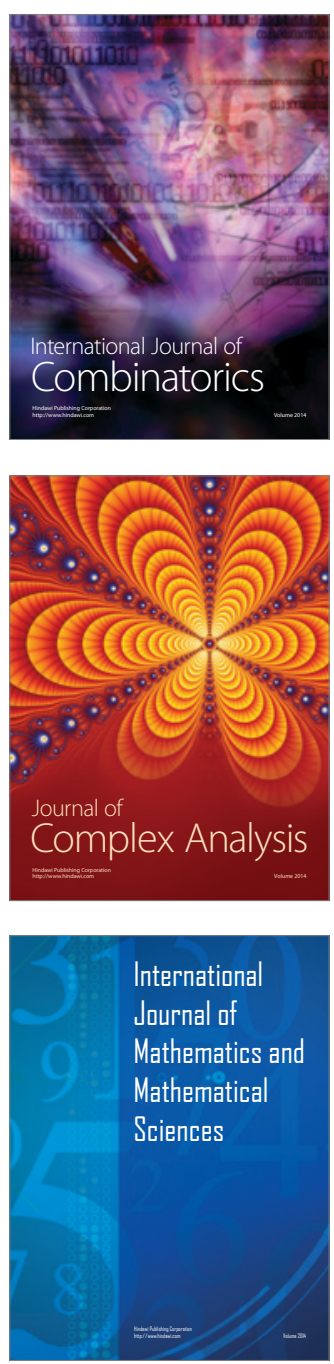
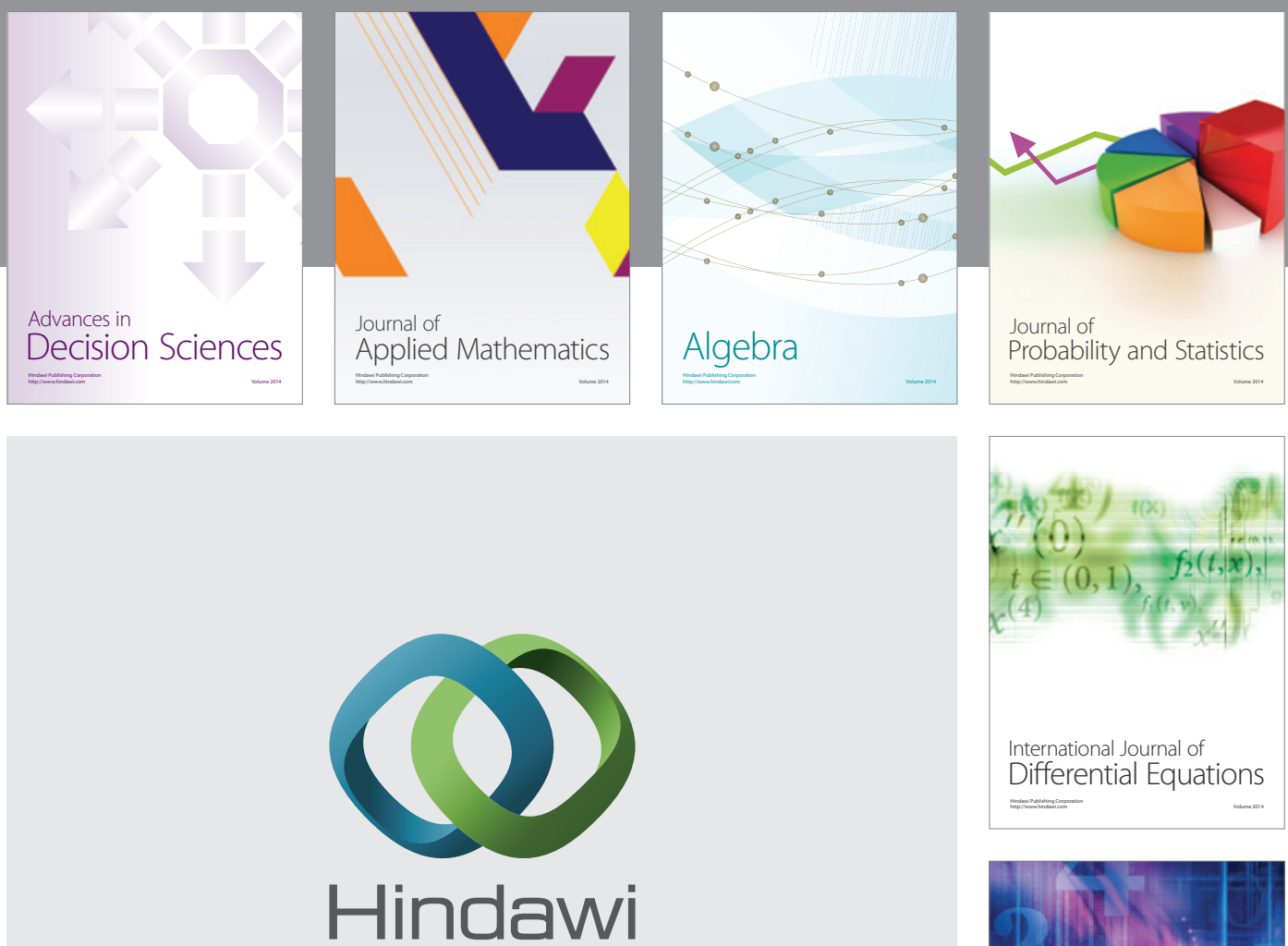

Submit your manuscripts at http://www.hindawi.com
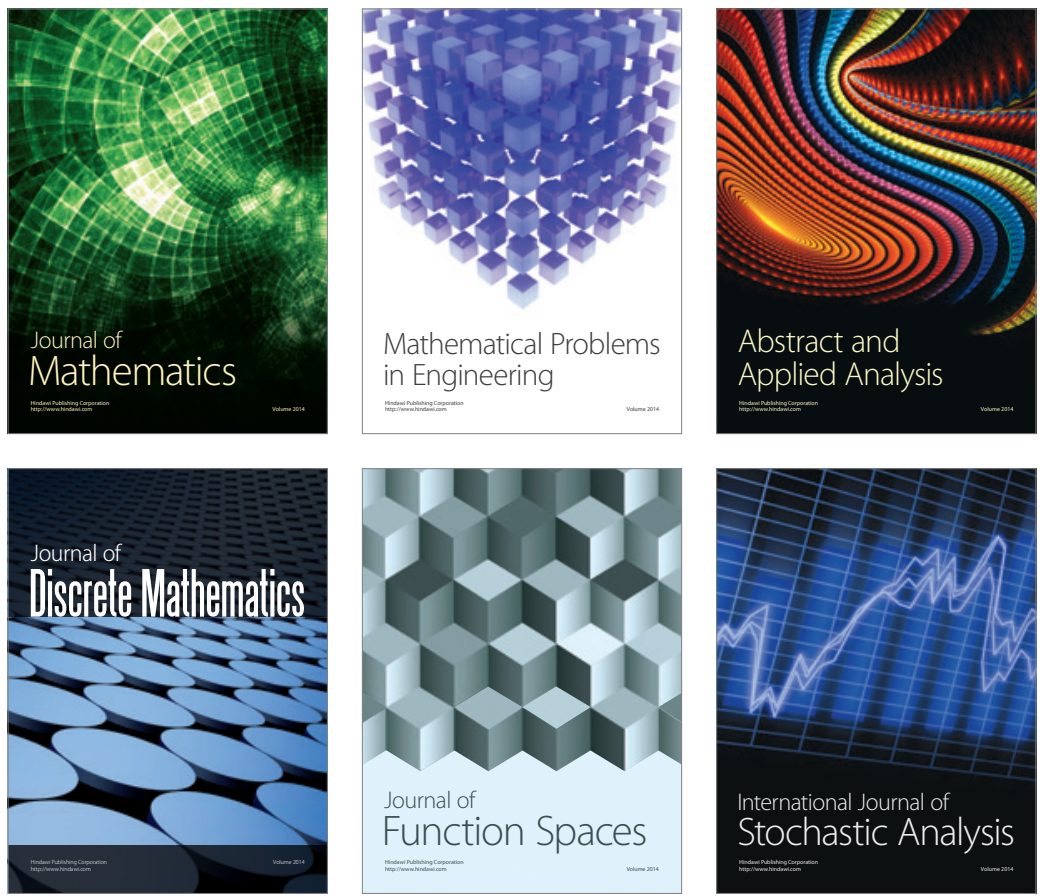

Journal of

Function Spaces

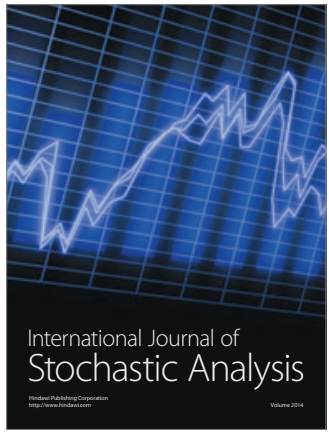

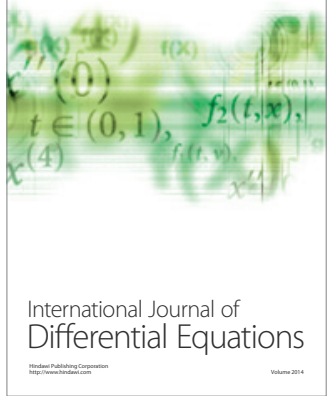
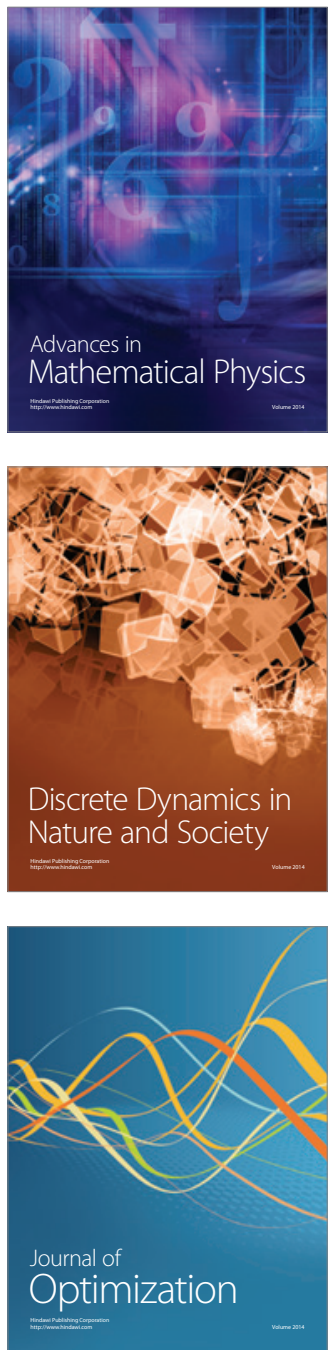